\title{
The CMS ECAL Calibration and Monitoring
}

\section{Mohammad Abrar Wadud*}

University of Minnesota

E-mail: wadudeumn . edu

Precise calibration and monitoring of the CMS electromagnetic calorimeter (ECAL) is a key ingredient in achieving the excellent ECAL performance required by many physics analyses employing electrons, photons and jets. This poster describes the methods used to monitor and intercalibrate the ECAL response, using physics channels such as W/Z boson decays to electrons, $\pi^{0}$ decays to photon pairs, and also exploiting the azimuthal symmetry of the minimum bias events. Results of the calibrations obtained with Run 2 data are presented.

European Physical Society Conference on High Energy Physics - EPS-HEP2019 -

10-17 July, 2019

Ghent, Belgium

${ }^{*}$ Speaker. 


\section{The Electromagnetic Calorimeter (ECAL)}

The ECAL is the primary instrument in CMS for measuring the energies of electrons and photons. It is homogeneous, hermetic, and composed of scintillating lead tungstate $\left(\mathrm{PbWO}_{4}\right)$ crystals: 61,200 in the barrel and 14,648 in two endcaps. Its pseudorapidity coverage is $|\eta|<1.479$ in the barrel and $1.479<|\eta|<3.0$ in the endcaps.

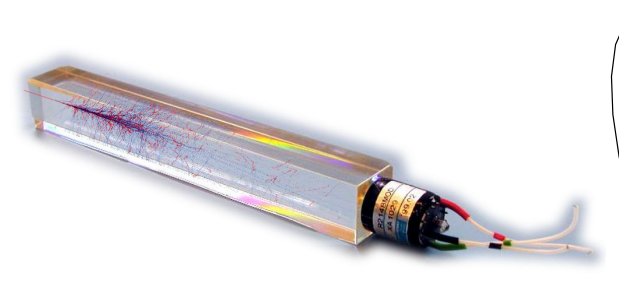

(a) An ECAL Endcap Crystal

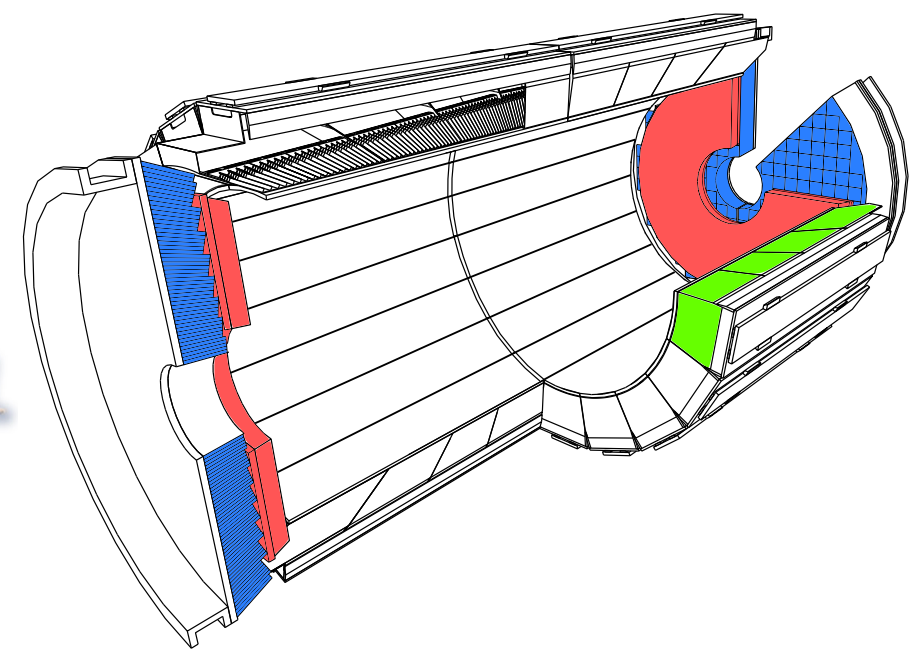

(b) Schematic of the ECAL

Figure 1

Figure 2 shows how the energy of an electron/photon is reconstructed from a cluster of crystals. The stability and uniformity of per-crystal response directly contribute to the ECAL energy resolution.

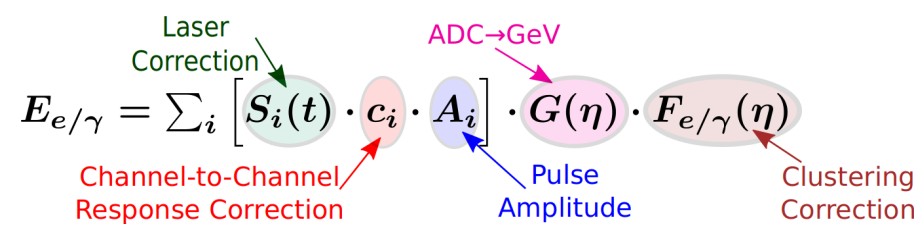

Figure 2: Corrections that are applied to reconstruct electron/photon energies

\section{Laser Corrections}

During data-taking, crystal response varies due to radiation-induced transparency loss. A laser monitoring system (Figure 4) records response variation during beam abort gaps and measures the corrections every 40 minutes (Figure 4). The response of a crystal to a reference laser is used to determine the correction with an empirical power law:

$$
\underset{\text { Correction }}{\frac{S(t)}{S_{0}}}=\underbrace{\left.\frac{R(t)}{R_{0}}\right)^{\alpha}}_{\text {Laser Response }}
$$

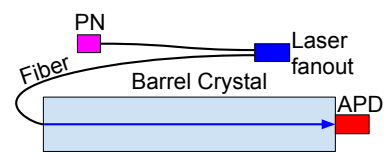

Figure 3: Laser Monitoring System 


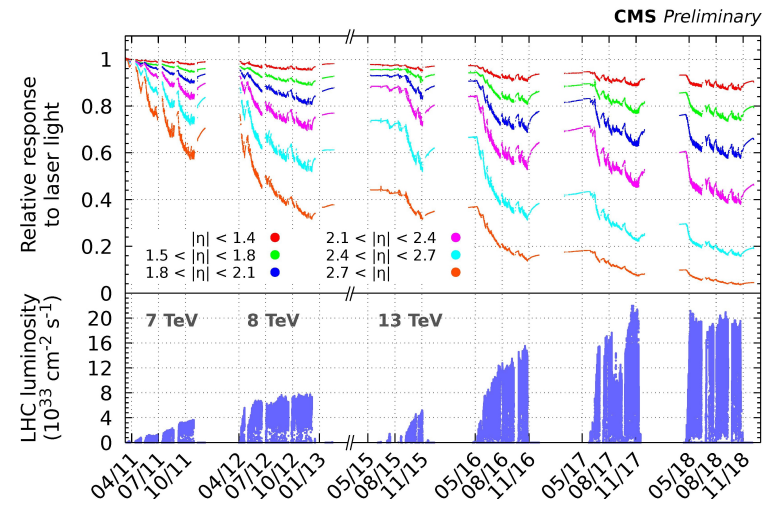

Figure 4: Relative response to laser light (440 nm in 2011 and $447 \mathrm{~nm}$ from 2012 onwards) injected in the ECAL crystals, measured by the ECAL laser monitoring system, averaged over all crystals in bins of pseudorapidity $(\eta)$, for the 2011, 2012, 2015, 2016, 2017 and 2018 data taking periods, with magnetic field at $3.8 \mathrm{~T}$. The response change observed in the ECAL channels is up to $13 \%$ in the barrel and it reaches up to $62 \%$ at $\eta \sim 2.5$, the limit of the tracker acceptance. The response change is up to $96 \%$ in the region closest to the beam pipe. The recovery of the crystal response during the periods without collisions is visible. These measurements, performed every 40 minutes, are used to correct the physics data.

\section{Monitoring}

The performance of the ECAL is monitored using reference physics events (Figure 5):

- $\pi^{0}$ invariant mass reconstructed in the two photon decay channel. This allows monitoring of low-energy reconstruction.

- Reconstructed ECAL energy $\left(E^{\mathrm{ECAL}}\right)$ of high-energy electrons from $\mathrm{W} \rightarrow e v$ and $Z \rightarrow e e$ events is compared with trackerreconstructed momentum $\left(p^{\mathrm{Tkr}}\right)$ to monitor the stability of $E^{\mathrm{ECAL}} / p^{\mathrm{Tkr}}$.

- High energy $e^{+} e^{-}$pairs from $Z$ decay is used to monitor the stability reconstructed $Z$ mass.

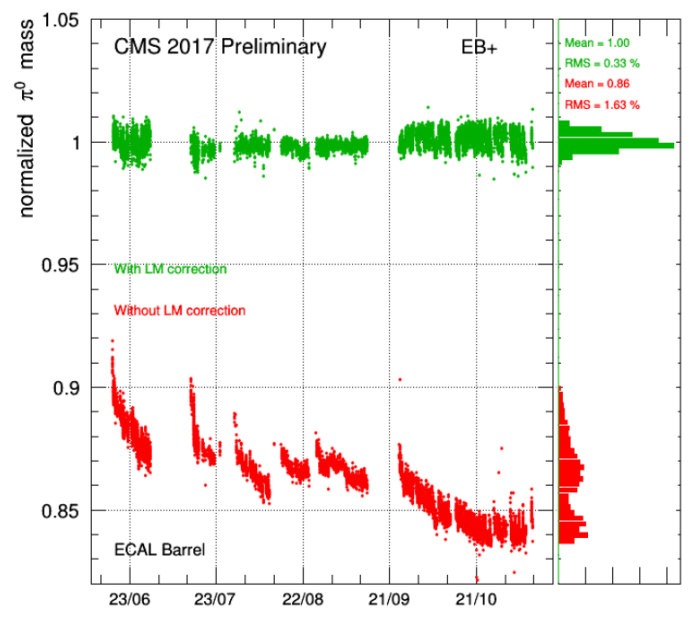

(a) Monitoring of $\pi^{0}$ Invariant Mass

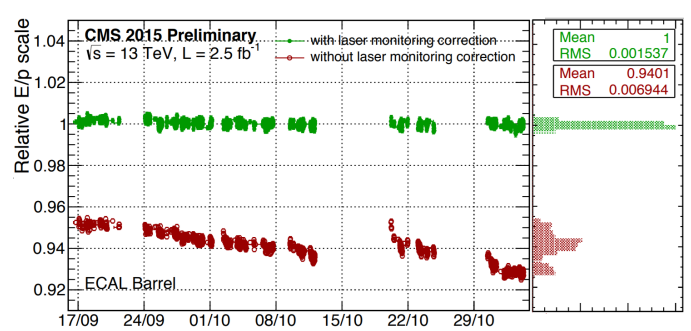

(b) Monitoring of $E^{\mathrm{ECAL} / p^{\mathrm{Tkr}}}$

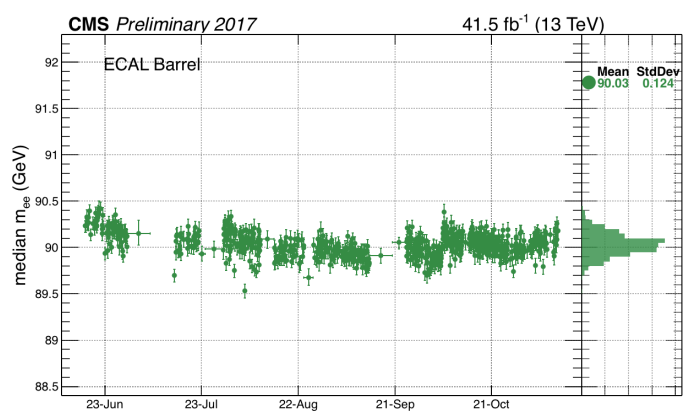

(c) Monitoring of Z Invariant Mass 


\section{Equalizing Channel-to-Channel Response}

Crystal response across ECAL is equalized by optimizing inter-calibration (IC) constants based on multiple physics techniques:

- $\phi$-symmetry: For a large sample of minimum bias events, the total deposited transverse energy should be the same on average in all crystals in a given $\eta$-ring.

- $\pi^{0} \rightarrow \gamma \gamma$ peak: Reconstructed peak from $\pi^{0} \rightarrow \gamma \gamma$ events is used to iteratively correct the IC for each channel.

- $Z \rightarrow e e$ peak: $Z$ peak is reconstructed from $e^{+} e^{-}$pairs by fitting $m_{e e}$ to a convolution of Breit-Wigner and Gaussian functions; a maximum-likelihood algorithm calculates the ICs.

- $E^{\mathrm{ECAL}} / p^{\mathrm{Tkr}}$ : The ratio of ECAL energy and tracker momentum for high energy electrons from $Z$ and $W$ decays is required to fit a data-based template ( 1 on average).

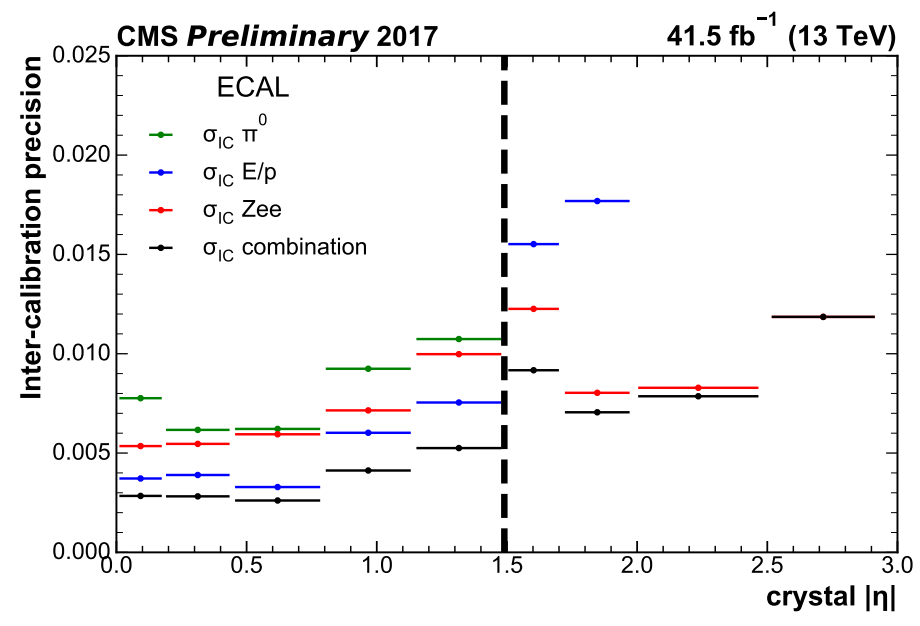

Figure 6: Combination of Intercalibration Techniques

Combination Each method is weighted by its energy resolution performance as measured in $Z \rightarrow$ ee decays to calculate the combined inter-calibration constants (Figure 6).

\section{Refinement of Run II Data}

The $139 \mathrm{fb}^{-1}$ of data collected in Run II (2015-2018) is being reprocessed to optimize the resolution and stability of the ECAL energy reconstruction. The plot in Figure 7 shows the relative resolution (with $Z$ electrons) in bins of $\eta$ for preliminary 2017 data (black) and the refined data (blue). The energy resolution performance is significantly improved with the refined reconstruction both in the barrel and in the endcaps calorimeters. 


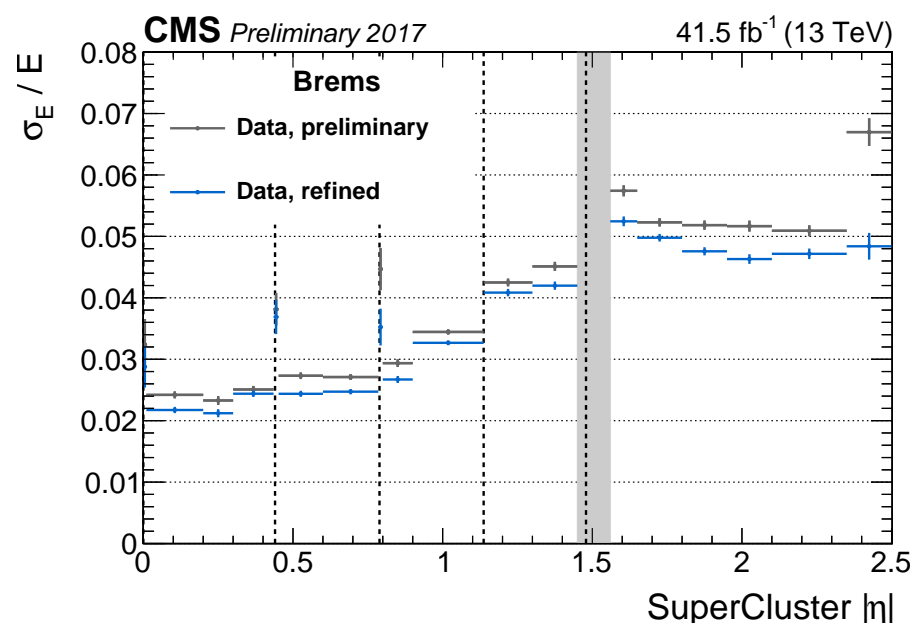

Figure 7: Effect of the refined calibration on the energy-resolution performance in 2017 Data

\section{References}

[1] The CMS Collaboration. Energy calibration and resolution of the CMS electromagnetic calorimeter in pp collisions at $\sqrt{s}=7$ TeV JINST 8.09 (2013), P09009-P09009

[2] The CMS Collaboration. Performance of photon reconstruction and identification with the CMS detector in proton-proton collisions at $\sqrt{s}=8 \mathrm{TeV}$, JINST 10.08 (2015) P08010-P08010

[3] The CMS Collaboration. Performance of electron reconstruction and selection with the CMS detector in proton-proton collisions at $\sqrt{s}=8 \mathrm{TeV}$, JINST 10.06 (2015) P06005-P06005 